A. GÜLSEN ${ }^{1,2}$, U. JAPPE ${ }^{1,2}$

\title{
Lipid transfer protein sensitization in an apple-allergic patient: a case report from Northern Europe
}

${ }^{1}$ Division of Clinical and Molecular Allergology, Research Center Borstel, Airway Research Center North (ARCN), member of the German Center for Lung Research, Borstel, Germany

${ }^{2}$ Interdisciplinary Allergy Outpatient Clinic, Department of Pneumology, University of Luebeck, Germany

\section{KeY WORDS}

apple; anaphylaxis; exercise-induced; FDEIA; lipid transfer protein

\author{
Corresponding address \\ Askin Gülsen \\ Research Center Borstel \\ Leibniz Lung Center \\ Parkallee 35, 23845 Borstel, Germany \\ Phone: +4945371887406 \\ Fax: +4945371883410 \\ E-mail: askingulsen@hotmail.com
}

Doi

10.23822/EurAnnACI.1764-1489.63

\begin{abstract}
Summary
We describe a case of a woman who developed three separate episodes of urticaria and anaphylaxis during exercise after consuming an apple, with immunological evidence that nonspecific lipid transfer proteins (LTP) may have been responsible for these reactions. LTP sensitivity can cause life-threatening allergies and anaphylaxis. LTP sensitization is common in Mediterranean countries. The knowledge is growing with the frequency of diagnoses in Northern Europe. Despite the geographic differences, LTP allergy should be kept on sight when facing severe anaphylaxis after consuming LTP-containing food.
\end{abstract}

\section{Introduction}

Nonspecific lipid transfer proteins (LTPs) are common in plants, widely distributed throughout different species, and they are one major cause of food allergy, especially in the Mediterranean region (1). The most clinically important LTP in this region is found especially in peach (Pru p 3) (2). Outside of Mediterranean areas, Pru p 3 associated with mugworth allergy was reported as a major allergen (3). Although reports on LTPs as the causative agents for food allergy in Northern and Eastern Europe are rare, observations in clinical practice to this regard are increasing in number. In these areas, predominantly birch pollen (Bet $\mathrm{v}$ 1) homologous allergens induced mild oropharyngeal reactions and individual cases of LTP sensitization have been described, for example, hazelnut (Cor a 8), apple (Mal d 3), peanut (Ara h 9), wheat (Tri a 14), cherry (Pru av 3), kiwi (Act d 10) and celery (Api g 6) (4). However, a very rare case of anaphylactic reaction to dragon fruit LTP was reported in recent years from Northern Europe (5).
Nonspecific LTPs are small and basic proteins with four disulfide bonds found in plants, (pollen and plant-derived foods), and some fungi. The disulfide bonds play a protective role and provide resistance to heat and digestion in the gastrointestinal tract (6). Therefore, they often induce primary gastrointestinal sensitization.

Exercise-induced anaphylaxis is a potentially fatal disease in which an immunological condition (immediate type allergy) is triggered by mild to heavy exercise. When food is identified as the causative source of allergen, the respective clinical condition is referred to as food-dependent exercise-induced anaphylaxis (FDEIA) (7). The pathomechanism of FDEIA is currently not fully understood. One interesting theory suggests that changes in mucosal permeability induced by cofactors such as non-steroidal anti-inflammatory drugs, alcohol consumption, exercise, or a combination thereof can enhance allergen absorption via the mucous membranes, resulting in increased exposure of the mast cells to allergens (8). Other mechanisms proposed to explain this syndrome include increased skeletal muscle and 
splanchnic blood flow and increased gastrin-induced mediator release in the postprandial phase (9).

We describe a case of a woman who developed three separate episodes of urticaria and anaphylaxis during exercise after consuming an apple, with immunological evidence that nonspecific lipid transfer proteins (LTP) may have been responsible for these reactions.

\section{Case report}

A 40-year-old woman presented to the emergency department with generalized urticaria, pruritus, sweating, and facial angioedema of the lips and tongue. She had no significant past medical history (except depression) or allergies, and was taking only venlafaxine as a regular antidepressant medication for 6 years. She presented to the emergency department a second time after developing head tingling accompanied by dizziness, swelling of the face, and sweating while jogging in the forest. The previous evening, the patient had drunken beer and eaten goulash with beef. The next morning, she had eaten an apple and buttermilk, and started exercise. The symptoms developed approximately 120 minutes after consuming the apple during exercise. Finally, she presented to the emergency department a third time after developing generalized urticaria and mild angioedema: The patient had gotten up, eaten breakfast (dark bread with cheese) and apple. After that, she went running and developed an allergic reaction.

Diagnostic allergy testing: serum total immunoglobulin E ( $\operatorname{IgE})$ was normal $(67.1 \mathrm{kU} / \mathrm{L})$. Skin prick testing revealed sensitization to all components of apple with a reaction diameter of 9 $\mathrm{mm}$. She was also sensitized to walnut $(5 \mathrm{~mm})$, celery $(4 \mathrm{~mm})$, anise $(4 \mathrm{~mm})$, kiwi fruit $(4 \mathrm{~mm})$ and chamomile $(4 \mathrm{~mm})$, but did not have symptoms of allergic rhinitis or oral allergy syndrome in her history. The positive control (histamine) was 7 $\mathrm{mm}$. While she was waiting in the outpatient clinic, the patient had eaten an apple in a resting state before the prick test was performed, because she did not at all assume apple to be the causative, and this accidental "open food challenge test" was tolerated well without exercise.

Investigation of specific IgE-antibodies to allergen sources and single allergens using ImmunoCAP (Immuno Solid-phase Allergen Chip; Phadia, Uppsala, Sweden) revealed a moderate sensitization to nonspecific LTPs from apple (Mal d 3) and peach (Pru p 3) as well as a low sensitization to peanut (Ara h 9), hazelnut (Cor a 8), and wheat (Tri a 14). She was not sensitized to birch (Bet v 1), or any of the storage proteins, profilins, or PR10 proteins included as potentially causative allergens for severe allergic reactions. In addition, we searched and found no sensitization to Gal-alpha-1.3-Gal Thyroglobulin. The positive and negative results are shown in table I. The provocation under exercise was not performed due to high risk of anaphylaxis. The synopsis of the patient's history, in vivo- and in vitro-tests led to the diagnosis of a FDEIA to apple due to the LTP sensitization. The patient was advised to avoid the consumption of fruits of the Rosaceae family (peach, apple, apricot, plum, cherry, and pear). We also recommended to especially observe the consumption of food in connection with physical exertion and alcohol consumption, as well as the intake of non-steroidal anti-inflammatory drugs. An adrenaline auto-injector, oral cetirizine, and prednisolone were prescribed, and the patient was provided with an anaphylaxis action plan. Since she was avoiding the consumption of apples, there was no re-presentation to the emergency department.

Comparison of the protein sequences of LTP from apple (Malus domesticus, Mal d 3) with the sequences of LTPs from other food allergen sources

We used www.allergen.org and the NCBI Database to compare the protein sequences in the identified allergens. The protein sequence of apple LTP (Mal d 3) showed 80.22\% to $86.81 \%$ similarity with LTPs from other Rosaceae fruits. The protein sequences of nut LTPs showed only $61.54 \%$ to $68.13 \%$ similarity

Table I - In vitro allergy diagnostic test: specific IgE-antibody detection results (ImmunoCAP).

\begin{tabular}{cccc}
\hline allergen source & allergen component & IgE-concentration (kU/l) & RAST-class \\
\hline apple & apple extract & 2.80 & 2 \\
\hline apple (Malus domesticus) $(\mathrm{NsLTP})$ & rMal d 3 & 13.10 & 3 \\
\hline peach (Prunus persica) $(\mathrm{NsLTP})$ & rPru p 3 & 6.25 & 3 \\
\hline peanut (Arachis hypogaea) $(\mathrm{NsLTP})$ & rAra h 9 & 0.67 & 1 \\
\hline hazelnut (Corylis avellana) $(\mathrm{NsLTP})$ & rCor a 8 & 0.53 & 1 \\
\hline wheat (Triticum aestivum) $(\mathrm{NsLTP})$ & rTri a 14 & 0.36 & 1 \\
\hline
\end{tabular}

Negative results to the following allergen components: Bet v 1 (the major birch pollen allergen); Gal-alpha-1.3-Gal thyroglobulin (red meat allergen); rAra h 1, rAra h 2, rAra h 3 (the storage proteins of peanut); rTri a 19 (wheat allergen); Cor a 9 (the storage protein of hazelnut); nGly $\mathrm{m} 5$ (the storage proteins of soybean); Api g 1 (PR-10 protein of celery); alpha lactalbumin, beta lactoglobulin and casein (milk); rye; sesame scrap; rice, mustard. 
with Mal d 3. The longest peptide in the protein sequences of the fruits that was similar between several fruit LTPs was between 20 and 31 amino acids (GGAVPPACCNGI). We consider that this protein segment may play an important role in the cross-reactions of fruits (table II).

\section{Discussion}

LTP sensitization with FDEIA is a rare disorder in which urticaria or anaphylaxis occurs during or after exercise and consumption of foods (mostly Rosaceae fruits). The symptoms may include erythema, rash, itching, dyspnea, nausea, flushing, diarrhea, and abdominal cramps. The symptoms may vary from mild to severe life-threatening anaphylactic reactions if the physical activity continues, including facial angioedema, laryngeal edema, sudden hypotension, and, as a result, cardiovascular collapse. Discontinuation of physical activity usually causes rapid improvement of the symptoms. Further external triggers include alcohol consumption, hot or cold temperatures, drugs (e.g., non-steroidal anti-inflammatory drugs such as aspirin), humidity, seasonal changes, lack of sleep, familial background, psychological stress, and certain phases of the menstrual cycle $(10,11)$. The prognosis and long-term follow-up of FDEIA have not been well described.

In our case, apple-dependent exercise-induced anaphylaxis was demonstrated. Our patient showed IgE-mediated moderate sensitization to apple and peach, and low sensitization to peanut, hazelnut, and wheat non-specific LTPs; however, there was no clinical relevance or history of allergic reactions except to apple. The observed apple-dependent, non-specific LTP-mediated, immediate-hypersensitivity-type reaction would be subthreshold at rest; however, because of the influence of exercise on mast cell releasability, it became clinically overt. We consider that, in our case, the established cofactors (exercise and alcohol) played an important role in the development of urticaria and anaphylaxis. However, environmental factors such as cold temperature and other non-immunologic factors may have also contributed to the increased mediator release.

Table II - Results of a sequence alignment of LTP sequences from different sources.

\begin{tabular}{cccccc}
\hline LTP & $\mathbf{0 - 1 0}$ & $\mathbf{1 1 - 2 0}$ & $\mathbf{2 1 - 3 0}^{1}$ & $\mathbf{3 1 - 4 0}$ & $\mathbf{4 1 - 5 0}^{-\mathbf{4 0}}$ \\
\hline apple (Mal d 3) & ITCGQVTSSL & APCIGYVRSG & GAVPPACCNG & IRTINGLART & TADRQTACNC \\
apricot (Pru ar 3) & ITCGQVSSSL & APCIGYVRGG & GAVPPACCNG & IRNVNNLART & TPDRRTACNC \\
pear (Pyr c 3) & ITCSQVSANL & APCINYVRSG & GAVPPACCNG & IKTINGLAKT & TPDRQAACNC \\
plum (Pru d 3) & ITCGQVSSNL & APCINYVKGG & GAVPPACCNG & IRNVNNLART & TADRRAACNC \\
cherry (Pru av 3) & LTCGQVSSNL & APCIAYVRGG & GAVPPACCNG & IRNINNLAKT & TADRQTACNC \\
peach (Pru p 3) & ITCGQVSSAL & APCIPYVRGG & GAVPPACCNG & IRNVNNLART & TPDRQAACNC \\
peanut (Ara h 9) & ISCGQVNSAL & APCIPFLTKG & GAPPPACCSG & VRGLLGALRT & TADRQAACNC \\
walnut (Jug r 3) & ITCGQVASSV & GSCIGYLRGT & VPTVPPSCCN & GVKSLNKAAA & TTADRQAACE \\
hazelnut ( Cor a 8) & LTCPQIKGNL & TPCVLYLKNG & GVLPPSCCKG & VRAVNDASRT & TSDRQSACNC \\
\hline
\end{tabular}

\begin{tabular}{cccccc}
\hline LTP & $\mathbf{5 1 - 6 0}$ & $\mathbf{6 1 - 7 0}$ & $\mathbf{7 1 - 8 0}$ & $\mathbf{8 1 - 9 1}$ & \% identity \\
\hline apple (Mal d 3) & LKNLAGSISG & VNPNNAAGLP & GKCGVNVPYK & ISTSTNCATVK & 100 \\
apricot (Pru ar 3) & LKQLSGSISG & VNPNNAAALP & GKCGVNIPYK & ISASTNCATVK & 86.81 \\
pear (Pyr c 3) & LKNLAGSVSG & VNPGNAESLP & GKCGVNVPYK & ISTSTNCATVK & 85.71 \\
plum (Pru d 3) & LKQLSGSIPG & VNPNNAAALP & GKCGVNVPYK & ISASTNCATVK & 83.52 \\
cherry (Pru av 3) & LKQLSASVPG & VNANNAAALP & GKCGVNVPYK & ISPSTNCATVK & 82.42 \\
peach (Pru p 3) & LKQLSASVPG & VNPNNAAALP & GKCGVHIPYK & ISASTNCATVK & 80.22 \\
peanut (Ara h 9) & LKAAAGSLRG & LNQGNAAALP & GRCGVSIPYK & ISTSTNCATIKK & 68.13 \\
walnut (Jug r 3) & CLKKTSGSIP & GLNPGLAAGLP & GKCGVSVPYK & ISTSTNCKAVK & 68.13 \\
hazelnut (Cor a 8) & LKDTAKGIAG & LNPNLAAGLP & GKCGVNIPYK & ISPSTNCNNVK & 61.54 \\
\hline
\end{tabular}

${ }^{1}$ The longest peptide in the protein sequences of the fruits that was similar between several fruit LTPs was between 20 and 31 amino acids (GGAVPPACCNGI). 
In a study published by Pascal and colleagues in 2012, no correlation was found between LTP-specific IgE levels and the severity of an allergic reaction. In their research, the main suspected foods reported by LTP allergic patients were peach, lettuce, walnut, hazelnut, peanut, and green beans. In $40 \%$ of patients, cofactors were necessary to induce symptoms (2). The co-factors for our patient were alcohol and exercise. In another study conducted by Asero et al. in 2014, the higher level of IgE to peach LTP (Pru p 3) was found to be associated with the cross-reactions of other plant-derived LTPs (12). In our patient, a cross-reaction was observed with other food LTPs, such as peach, hazelnut, peanut and wheat, but it was clinically insignificant. Therefore, it was thought that cross-sensitization did not fully reflect the clinical condition, but can be helpful to determine a diagnosis. In addition, similar sequential epitopes of LTPs may play an important role to the cross-sensitization.

Moreover, some cases in the literature developed allergic symptoms in the following period only by intake of foods containing heated apple, without exercise (13). In some countries such as Spain, sublingual immunotherapies are currently available for severely allergic patients, with the aim to increase the provocation threshold (6). A large number of foods have already partially been described on a molecular level, defining major allergens and the respective protein families, and the list is still growing. Although, the reason for the observed geographical distribution and differences in LTP sensitivity is not fully understood, the nutrition habits, a genetic predisposition, and differences of pollen exposure may play an important role (14).

\section{Conclusion}

LTP sensitivity can cause life-threatening allergies and anaphylaxis. Although LTP allergy is common mainly in Mediterranean countries, the number of cases is increasing in Northern Europe. Despite the geographic differences, LTP allergy should be considered when facing severe anaphylaxis after consuming LTP-containing food.

\section{Conflict of Interest}

The authors declare that they have no conflict of interest.

\section{References}

1. Asero R, Antonicelli L, Arena A, et al. EpidemAAITO: features of food allergy in Italian adults attending allergy clinics: a multicentre study. Clin Exp Allergy 2009; 39:547-555.

2. Pascal M, Muñoz-Cano R, Reina Z, et al. Lipid transfer protein syndrome: clinical pattern, cofactor effect and profile of molecular sensitization to plant-foods and pollens. Clin Exp Allergy 2012; 42:1529-1539.

3. Gao ZS, Yang ZW, Wu SD,et al. Peach allergy in China: a dominant role for mugwort pollen lipid transfer protein as a primary sensitizer. J Allergy Clin Immunol 2013; 131(1):224-6.e1-3.

4. Petersen A, Kleine-Tebbe J, Scheurer S. Stable plant food allergens I: Lipid-Transfer-Proteins. In: Kleine-Tebbe J, Jakob T, editors. Molecular allergy diagnostics: innovation for a better patient management. Cham: Springer International Publishing 2017; 60-62.

5. Kleinheinz A, Lepp U, Hausen BM, Petersen A, Becker WM. Anaphylactic reaction to (mixed) fruit juice containing dragon fruit. J Allergy Clin Immunol 2009; 124(4):841.

6. Gomez F, Bogas G, Gonzalez M, et al. The clinical and immunological effects of Pru p 3 sublingual immunotherapy on peach and peanut allergy in patients with systemic reactions. Clin Exp Allergy 2017; 47:339-350.

7. Giacco SRD. Exercise-induced anaphylaxis: an update. Breathe 2012; 8(4):299-306.

8. Lambert GP, Boylan M, Laventure JP, Bull A, Lanspa S. Effect of aspirin and ibuprofen on GI permeability during exercise. Int J Sports Med 2007; 28:722-726.

9. Castells MC, Horan RF, Sheffer AL. Exercise-induced anaphylaxis (EIA). Clin Rev Allergy Immunol 1999; 17(4):413-424.

10. Sheffer AL, Austen KF. Exercise-induced anaphylaxis. J Allergy Clin Immunol 1980; 66:106-111.

11. Barg W, Medrala W, Wolanczyk-Medrala A. Exercise-Induced Anaphylaxis: An Update on Diagnosis and Treatment. Current Allergy and Asthma Reports 2011; 11(1):45-51.

12. Asero R. In patients with LTP syndrome food-specific IgE show a predictable hierarchical order. Eur Ann Allergy Clin Immunol 2014; 46:142-146.

13. Kaneko M, Yagi H, Koyama $\mathrm{H}$ et al. A case of apple allergy with initial symptoms like food- dependent exercise-induced anaphylaxis. Arerugi 2013; 62(6):698-703.

14. Schocker F, Lüttkopf D, Scheurer S, et al. Recombinant lipid transfer protein Cor a 8 from hazelnut: a new tool for in vitro diagnosis of potentially severe hazelnut allergy. J Allergy Clin Immunol 2004; 113(1):141-147. 